\title{
Viscosity Approximation Methods for Equilibrium Problems, Variational Inequality Problems of Infinitely Strict Pseudocontractions in Hilbert Spaces
}

\begin{abstract}
Aihong Wang
College of Science, Civil Aviation University of China, Tianjin 300300, China

Correspondence should be addressed to Aihong Wang, ahwang@cauc.edu.cn

Received 23 April 2012; Accepted 2 August 2012

Academic Editor: Hong-Kun Xu

Copyright (C) 2012 Aihong Wang. This is an open access article distributed under the Creative Commons Attribution License, which permits unrestricted use, distribution, and reproduction in any medium, provided the original work is properly cited.

We introduce an iterative scheme by the viscosity approximation method for finding a common element of the set of the solutions of the equilibrium problem and the set of fixed points of infinitely strict pseudocontractive mappings. Strong convergence theorems are established in Hilbert spaces. Our results improve and extend the corresponding results announced by many others recently.
\end{abstract}

\section{Introduction}

Let $H$ be a real Hilbert space and let $C$ be a nonempty convex subset of $H$.

A mapping $S$ of $C$ is said to be a $\kappa$-strict pseudocontraction if there exists a constant $\kappa \in[0,1)$ such that

$$
\|S x-S y\|^{2} \leq\|x-y\|^{2}+\kappa\|(I-S) x-(I-S) y\|^{2},
$$

for all $x, y \in C$; see [1]. We denote the set of fixed points $S$ by $F(S)$ (i.e., $F(S)=\{x \in C: S x=$ $x\})$.

Note that the class of strict pseudocontraction strictly includes the class of nonexpansive mappings which are mappings $S$ on $C$ such that

$$
\|S x-S y\| \leq\|x-y\|,
$$


for all $x, y \in C$. That is, $S$ is nonexpansive if and only if $S$ is a 0 -strict pseudocontraction. Let $\Phi$ be a bifunction from $C \times C$ to $\mathbb{R}$, where $\mathbb{R}$ is the set of real numbers. The equilibrium problem for $\Phi: C \times C \rightarrow \mathbb{R}$ is to find $x \in C$ such that

$$
\Phi(x, y) \geq 0, \quad \forall y \in C
$$

The set of solutions of (1.3) is denoted by $E P(\Phi)$. Given a mapping $B: C \rightarrow H$, let $\Phi(x, y)=$ $\langle B x, y-x\rangle$ for all $x, y \in C$. Then the classical variational inequality problem is to find $x \in C$ such that $\langle B x, y-x\rangle \geq 0$. We denote the solution of the variational inequality by $\operatorname{VI}(C, B)$; that is

$$
\operatorname{VI}(C, B)=\{x \in C:\langle B x, y-x\rangle \geq 0\}
$$

Let $A$ be a strongly positive linear-bounded operator on $H$ if there is a constant $\bar{\gamma}>0$ with property

$$
\langle A x, x\rangle \geq \bar{\gamma}\|x\|^{2}, \quad \forall x \in H .
$$

A typical problem is to minimize a quadratic function over the set of the fixed points a nonexpansive mapping on a real Hilbert space $H$ :

$$
\min _{x \in E} \frac{1}{2}\langle A x, x\rangle-\langle x, b\rangle
$$

where $A$ is a linear-bounded operator, $E$ is the fixed point set of a nonexpansive mapping $S$ on $H$, and $b$ is a given point in $H$. The problem (1.3) is very general in the sense that it includes, as special cases, optimization problems, variational inequalities, minimax problems, the Nash equilibrium problem in noncooperative games, and others; see [1-11]. In particular, Combettes and Hirstoaga [4] proposed several methods for solving the equilibrium problem. On the other hand, Mann [6], Shimoji and Takahashi [8] considered iterative schemes for finding a fixed point of a nonexpansive mapping. Further, Acedo and $\mathrm{Xu}$ [12] projected new iterative methods for finding a fixed point of strict pseudocontractions.

In 2006, Marino and Xu [7] introduced the general iterative method: for $x_{1}=x \in C$,

$$
x_{n+1}=\alpha_{n} \gamma f\left(x_{n}\right)+\left(I-\alpha_{n} A\right) T x_{n}, \quad n \geq 1 .
$$

They proved that the sequence $\left\{x_{n}\right\}$ of parameters satisfies appropriate condition and that the sequence $\left\{x_{n}\right\}$ generated by (1.7) converges strongly to the unique solution of the variational inequality $\langle(\gamma f-A) q, p-q\rangle \leq 0, p \in F(T)$. Recently, Liu [5] considered a general iterative method for equilibrium problems and strict pseudocontractions:

$$
\begin{gathered}
\Phi\left(u_{n}, y\right)+\frac{1}{r_{n}}\left\langle y-u_{n}, u_{n}-x_{n}\right\rangle \geq 0, \quad \forall y \in C, \\
y_{n}=\beta_{n} u_{n}+\left(1-\beta_{n}\right) S u_{n}, \\
x_{n+1}=\varepsilon_{n} \gamma f\left(x_{n}\right)+\left(I-\varepsilon_{n} A\right) u_{n}, \quad \forall n \geq 1
\end{gathered}
$$


where $S$ is a $k$-strict pseudocondition mapping and $\left\{\varepsilon_{n}\right\},\left\{\beta_{n}\right\}$ are sequences in $(0,1)$. They proved that under certain appropriate conditions over $\left\{\varepsilon_{n}\right\},\left\{\beta_{n}\right\}$, and $\left\{r_{n}\right\}$, the sequences $\left\{x_{n}\right\}$ and $\left\{u_{n}\right\}$ both converge strongly to some $q \in F(S) \cap E P(\Phi)$, which solves some variational inequality problems. Tian [10] proposed a new general iterative algorithm: for nonexpansive mapping $T: H \rightarrow H$ with $F(T) \neq \phi$,

$$
x_{n+1}=\alpha_{n} \gamma f\left(x_{n}\right)+\left(I-\mu \alpha_{n} F\right) T x_{n}, \quad \forall n \geq 1,
$$

where $F$ is a $k$-Lipschitzian and $\eta$-strong monotone operator. He obtained that the sequence $x_{n}$ generated by (1.9) converges to a point $q$ in $F(T)$, which is the unique solution of the variational inequality $\langle(\gamma f-A) q, p-q\rangle \leq 0, p \in F(T)$. Very recently, Wang [13] considered a general composite iterative method for infinite family strict pseudocontractions: for $x_{1}=x \in$ C,

$$
\begin{gathered}
y_{n}=\beta_{n} x_{n}+\left(1-\beta_{n}\right) W_{n} x_{n}, \\
x_{n+1}=\alpha_{n} \gamma f\left(x_{n}\right)+\left(I-\mu \alpha_{n} F\right) y_{n}, \quad \forall n \geq 1,
\end{gathered}
$$

where $W_{n}$ is a mapping defined by (2.5), $F$ is a $k$-Lipschitzian, and $\eta$-strongly monotone operator. With some appropriate condition, the sequence $\left\{x_{n}\right\}$ generated by (1.10) converges strongly to a common element of the fixed point of an infinite family of $\lambda_{i}$-strictly pseudocontractive mapping, which is a unique solution of the variational inequality $\langle(\gamma f-$ A) $q, p-q\rangle \leq 0, p \in F(T)$. Kumam proposed many algorithms for the equilibrium and the fixed point problems with $k$-strict pseudoconditions; see [14-16]. In particular, in 2011, Kumam and Jaiboon [14] considered a system of mixed equilibrium problems, variational inequality problems, and strict pseudocontractive mappings:

$$
\begin{aligned}
& x_{1} \in E, \quad u_{n} \in E, \quad v_{n} \in E, \\
& u_{n}=T_{r}^{\phi_{1}, \varphi} x_{n}, \\
& v_{n}=T_{s}^{\phi_{2}, \varphi} x_{n}, \\
& z_{n}=P_{E}\left(u_{n}-\mu_{n} C u_{n}\right), \\
& y_{n}=P_{E}\left(v_{n}-\lambda_{n} C v_{n}\right), \\
& k_{n}=a_{n} S_{k} x_{n}+b_{n} y_{n}+c_{n} z_{n}, \\
& x_{n+1}=\varepsilon_{n} \gamma f\left(x_{n}\right)+\beta_{n} x_{n}+\left(\left(1-\beta_{n}\right) I-\varepsilon_{n} A\right) k_{n}, \quad \forall n \geq 1,
\end{aligned}
$$

where $S$ is a $k$-strict pseudocondition mapping. They proved that under certain appropriate conditions over $\left\{\varepsilon_{n}\right\},\left\{\beta_{n}\right\},\left\{r_{n}\right\},\left\{a_{n}\right\},\left\{b_{n}\right\},\left\{c_{n}\right\},\left\{\lambda_{n}\right\}$, and $\left\{\mu_{n}\right\}$, the sequence $\left\{x_{n}\right\}$ converges strongly to a point $q \in \Theta$ which is the unique solution of the variational inequality $\langle(A-$ $\gamma f) q, x-q\rangle \geq 0$. Inprasit [17] proposed a viscosity approximation methods to solving the generalized equilibrium and fixed point problems of finite family of nonexpansive mapping in Hilbert spaces. 
In this paper, motivated by the above facts, we use the viscosity approximation method to find a common element of the set of solutions of the equilibrium problem $\operatorname{VI}(C, B)$ and the set of fixed points of a infinite family of strict pseudocontractions.

\section{Preliminaries}

Throughout this paper, we always write $\rightarrow$ for weak convergence and $\rightarrow$ for strong convergence. We need some facts and tools in a real Hilbert space $H$ which are listed as below.

Lemma 2.1. Let $H$ be a real Hilbert space. There hold the following identities:

(i) $\|x-y\|^{2}=\|x\|^{2}-\|y\|^{2}-2\langle x-y, y\rangle, \forall x, y \in H$,

(ii) $\|t x+(1-t) y\|^{2}=t\|x\|^{2}+(1-t)\|y\|^{2}-t(1-t)\|x-y\|^{2}, \forall t \in[0,1], \forall x, y \in H$.

Lemma 2.2 (see [18]). Assume that $\left\{\alpha_{n}\right\}$ is a sequence of nonnegative real numbers such that

$$
\alpha_{n+1} \leq\left(1-\rho_{n}\right) \alpha_{n}+\sigma_{n}
$$

where $\left\{\rho_{n}\right\}$ is a sequence in $(0,1)$ and $\left\{\sigma_{n}\right\}$ is a sequence such that

(i) $\sum_{n=1}^{\infty} \rho_{n}=\infty$,

(ii) $\lim \sup _{n \rightarrow \infty}\left(\sigma_{n} / \rho_{n}\right) \leq 0$ or $\sum_{n=1}^{\infty}\left|\sigma_{n}\right|<\infty$.

Then $\lim _{n \rightarrow \infty} a_{n}=0$.

Recall that given a nonempty closed convex subset $C$ of a real Hilbert space $H$, for any $x \in H$, there exists a unique nearest point in $C$, denoted by $P_{C} x$, such that

$$
\left\|x-P_{C} x\right\| \leq\|x-y\|
$$

for all $y \in C$. Such a $P_{C}$ is called the metric (or the nearest point) projection of $H$ onto $C$. As we all know, $y=P_{C} x$ if and only if there holds the relation:

$$
\langle x-y, y-z\rangle \geq 0 \quad \forall z \in C
$$

Lemma 2.3 (see [13]). Let $A: H \rightarrow H$ be an L-Lipschitzian and $\eta$-strongly monotone operator on a Hilbert space $H$ with $L>0, \eta>0,0<\mu<2 \eta / L^{2}$, and $0<t<1$. Then $S=(I-t \mu A): H \rightarrow H$ is a contraction with contractive coefficient $1-t \tau$ and $\tau=(1 / 2) \mu\left(2 \eta-\mu L^{2}\right)$.

Lemma 2.4 (see [1]). Let $S: C \rightarrow C$ be a $\kappa$-strict pseudocontraction. Define $T: C \rightarrow C$ by $T x=\lambda x+(1-\lambda)$ Sx for each $x \in C$. Then, as $\lambda \in[\kappa, 1), T$ is a nonexpansive mapping such that $F(T)=F(S)$. 
Lemma 2.5 (see [10]). Let $H$ be a Hilbert space and $f: H \rightarrow H$ a contraction with coefficient $0<\alpha<1$, and $A: H \rightarrow H$ an L-Lipschitzian continuous operator and $\eta$-strongly monotone with $L>0, \eta>0$. Then for $0<\gamma<\mu \eta / \alpha$,

$$
\left\langle x-y_{,}(\mu A-\gamma f) x-(\mu A-\gamma f) y\right\rangle \geq(\mu \eta-\gamma \alpha)\|x-y\|^{2}, \quad x, y \in H
$$

That is, $\mu A-\gamma f$ is strongly monotone with coefficient $\mu \eta-\gamma \alpha$.

Let $\left\{S_{n}\right\}$ be a sequence of $\kappa_{n}$-strict pseudo-concontractions. Define $S_{n}^{\prime}=\theta_{n} I+(1-$ $\left.\theta_{n}\right) S_{n}, \theta_{n} \in\left[\mathcal{K}_{n}, 1\right)$. Then, by Lemma 2.4, $S_{n}^{\prime}$ is nonexpansive. In this paper, we consider the mapping $W_{n}$ defined by

$$
\begin{aligned}
& U_{n, n+1}=I, \\
& U_{n, n}=t_{n} S_{n}^{\prime} U_{n, n+1}+\left(1-t_{n}\right) I, \\
& U_{n, n-1}=t_{n-1} S_{n-1}^{\prime} U_{n, n}+\left(1-t_{n-1}\right) I, \\
& \cdots, \\
& U_{n, i}=t_{i} S_{i}^{\prime} U_{n, i+1}+\left(1-t_{i}\right) I, \\
& \cdots, \\
& U_{n, 2}=t_{2} S_{2}^{\prime} U_{n, 3}+\left(1-t_{2}\right) I, \\
& W_{n}=U_{n, 1}=t_{1} S_{1}^{\prime} U_{n, 2}+\left(1-t_{1}\right) I .
\end{aligned}
$$

Lemma 2.6 (see [8]). Let $C$ be a nonempty closed convex subset of a strictly convex Banach space $E$, let $S_{1}^{\prime}, S_{2}^{\prime}, \ldots$ be nonexpansive mappings of $C$ into itself such that $\cap_{i=1}^{\infty} F\left(S_{i}^{\prime}\right) \neq \emptyset$, and let $t_{1}, t_{2}, \ldots$ be real numbers such that $0<t_{i} \leq b<1$, for every $i=1,2, \ldots$. Then, for any $x \in C$ and $k \in N$, the limit $\lim _{n \rightarrow \infty} U_{n, k} x$ exists.

Using Lemma 2.6, one can define the mapping $W$ of $C$ into itself as follows:

$$
W x:=\lim _{n \rightarrow \infty} W_{n} x=\lim _{n \rightarrow \infty} U_{n, 1} x, \quad x \in C .
$$

Lemma 2.7 (see [8]). Let $C$ be a nonempty closed convex subset of a strictly convex Banach space $E$. Let $S_{1}^{\prime}, S_{2}^{\prime}, \ldots$ be nonexpansive mappings of $C$ into itself such that $\cap_{i=1}^{\infty} F\left(S_{i}^{\prime}\right) \neq \emptyset$, and let $t_{1}, t_{2}, \ldots$ be real numbers such that $0<t_{i} \leq b<1$, for all $i \geq 1$. If $K$ is any bounded subset of $C$, then

$$
\lim _{n \rightarrow \infty} \sup _{x \in K}\left\|W x-W_{n} x\right\|=0
$$

Lemma 2.8 (see [3]). Let $C$ be a nonempty closed convex subset of a Hilbert space $H$, let $\left\{S_{i}^{\prime}: C \rightarrow\right.$ $C\}$ be a family of infinite nonexpansive mappings with $\cap_{i=1}^{\infty} F\left(S_{i}^{\prime}\right) \neq \emptyset$, and let $t_{1}, t_{2}, \ldots$ be real numbers such that $0<t_{i} \leq b<1$, for every $i=1,2, \ldots$. Then $F(W)=\cap_{i=1}^{\infty} F\left(S_{i}^{\prime}\right)$. 
For solving the equilibrium problem, let us assume that the bifunction $\Phi$ satisfies the following conditions:

(A1) $\Phi(x, x)=0$ for all $x \in C$;

(A2) $\Phi$ is monotone; that is $\Phi(x, y)+\Phi(y, x) \leq 0$ for any $x, y \in C$;

(A3) for each $x, y, z \in C$, limsup $\sup _{t \rightarrow 0} \Phi(t z+(1-t) x, y) \leq \Phi(x, y)$;

(A4) $\Phi(x, \cdot)$ is convex and lower semicontinuous for each $x \in C$.

We recall some lemmas which will be needed in the rest of this paper.

Lemma 2.9 (see [2]). Let $C$ be a nonempty closed convex subset of $H$, let $\Phi$ be bifunction from $C \times C$ to $\mathbb{R}$ satisfying (A1)-(A4), and let $r>0$ and $x \in H$. Then there exists $z \in C$ such that

$$
\Phi(z, y)+\frac{1}{r}\langle y-z, z-x\rangle \geq 0, \quad \forall y \in C
$$

Lemma 2.10 (see [4]). Let $\phi$ be a bifunction from $C \times C$ into $\mathbb{R}$ satisfying (A1)-(A4). Then, for any $r>0$ and $x \in H$, there exists $z \in C$ such that

$$
\Phi(z, y)+\frac{1}{r}\langle y-z, z-x\rangle \geq 0, \quad \forall y \in C
$$

Further, if $T_{r} x=\{z \in C ; \Phi(z, y)+(1 / r)\langle y-z, z-x\rangle \geq 0, \forall y \in C\}$, then the following hold:

(1) $T_{r}$ is single-valued;

(2) $T_{r}$ is firmly nonexpansive;

(3) $F\left(T_{r}\right)=E P(\phi)$;

(4) $E P(\phi)$ is closed and convex.

Lemma 2.11 (see [9]). Let $\left\{x_{n}\right\}$ and $\left\{z_{n}\right\}$ be bounded sequences in a Banach space, and let $\left\{\beta_{n}\right\}$ be a sequence of real numbers such that $0<\liminf _{n \rightarrow \infty} \beta_{n} \leq \limsup _{n \rightarrow \infty} \beta_{n}<1$ for all $n=0,1,2, \ldots$. Suppose that $x_{n+1}=\left(1-\beta_{n}\right) z_{n}+\beta_{n} x_{n}$ for all $n=0,1,2, \ldots$ and $\lim \sup _{n \rightarrow \infty}\left\|z_{n+1}-z_{n}\right\|-\| x_{n+1}-$ $x_{n} \| \leq 0$. Then $\lim _{n \rightarrow \infty}\left\|z_{n}-x_{n}\right\|=0$.

Lemma 2.12 (see [11]). Let $C, H, F$, and $T_{r} x$ be as in Lemma 2.9. Then the following holds:

$$
\left\|T_{s} x-T_{t} x\right\|^{2} \leq\left\langle T_{s} x-T_{t} x, T_{s} x-x\right\rangle,
$$

for all $s, t>0$, and $x \in H$.

Lemma 2.13 (see [13]). Let $H$ be a Hilbert space, and let $C$ be a nonempty closed convex subset of $H$, and $T: C \rightarrow C$ a nonexpansive mapping with $F(T) \neq \emptyset$. If $\left\{x_{n}\right\}$ is a sequence in $C$ weakly converging to $x$ and if $\left\{(I-T) x_{n}\right\}$ converges strongly to $y$, then $(I-T) x=y$.

\section{Main Results}

Now we start and prove our main result of this paper. 
Theorem 3.1. Let $C$ be a nonempty closed convex subset of a real Hilbert space $H$. Let $\phi$ be a bifunction from $C \times C \rightarrow \mathbb{R}$ satisfying $(A 1)-(A 4)$. Let $S_{i}: C \rightarrow C$ be a family $\kappa_{i}$-strict pseudocontractions for some $0 \leq \kappa_{i}<1$. Assume the set $\Omega=\operatorname{VI}(C, B) \cap_{i=1}^{\infty} F\left(S_{i}\right) \cap E P(\phi) \neq \emptyset$. Let $f$ be a contraction of $H$ into itself with $\alpha \in(0,1)$, and let $A$ be a strongly positive linear bounded operator on $H$ with coefficient $\gamma>0$ and $0<\gamma<\bar{\gamma} / \gamma$. Let $B: C \rightarrow H$ be an $\xi$-inverse strongly monotone mapping. Let $W_{n}$ be the mapping generated by $S_{i}^{\prime}$ and $t_{i}$ as in (2.5). Let $\left\{x_{n}\right\}$ be a sequence generated by the following algorithm:

$$
\begin{gathered}
\phi\left(z_{n}, y\right)+\frac{1}{\lambda_{n}}\left\langle y-z_{n}, z_{n}-x_{n}\right\rangle \geq 0, \\
y_{n}=P_{C}\left(I-\mu_{n} B\right) z_{n}, \\
K_{n}=\alpha_{n} x_{n}+\left(1-\alpha_{n}\right) W_{n} y_{n}, \\
x_{n+1}=\varepsilon_{n} \gamma f\left(x_{n}\right)+\beta_{n} x_{n}+\left(\left(1-\beta_{n}\right) I-\varepsilon_{n} A\right) K_{n},
\end{gathered}
$$

where $\left\{\varepsilon_{n}\right\},\left\{\beta_{n}\right\},\left\{\alpha_{n}\right\}$, and $\left\{\lambda_{n}\right\}$ are sequences in $(0,1)$. Assume that the control sequences satisfy the following restrictions:

(i) $\lim _{n \rightarrow \infty} \varepsilon_{n}=0$ and $\Sigma_{n=1}^{\infty} \varepsilon_{n}=\infty$;

(ii) $0<\liminf _{n \rightarrow \infty} \beta_{n} \leq \lim \sup _{n \rightarrow \infty} \beta_{n}<1$;

(iii) $0<\lim _{n \rightarrow \infty}\left(\lambda_{n} / \lambda_{n+1}\right)=1$;

(iv) $\lim _{n \rightarrow \infty}\left|\alpha_{n+1}-\alpha_{n}\right|=0$;

(v) $0<\mu_{n} \leq 2 \xi$;

(vi) $\lim _{n \rightarrow \infty} \alpha_{n}=a$.

Then $\left\{x_{n}\right\}$ converges strongly to $q \in \Omega$ which is the unique solution of the variational inequality

$$
\langle(A-\gamma f) q, x-q\rangle \geq 0, \quad \forall x \in \Omega,
$$

or equivalent $q=P_{\Omega}(I-A+\gamma f)(q)$, where $P$ is a metric projection mapping form $H$ onto $\Omega$.

Proof. Since $\varepsilon_{n} \rightarrow 0$, as $n \rightarrow \infty$, we may assume, without loss of generality, that $\varepsilon_{n} \leq(1-$ $\left.\beta_{n}\right)\|A\|^{-1}$ for all $n \in \mathbb{N}$. By Lemma 2.3, we know that if $0 \leq \rho \leq\|A\|^{-1}$, then $\|I-\rho A\| \leq 1-\rho \bar{\gamma}$. We will assume that $\|I-A\| \leq 1-\bar{\gamma}$. Since $\mathrm{A}$ is a strongly positive bounded linear operator on $H$, we have

$$
\|A\|=\sup \{|\langle A x, x\rangle|: x \subseteq H,\|x\|=1\} .
$$

Observe that

$$
\begin{aligned}
\left\langle\left(\left(1-\beta_{n}\right) I-\varepsilon_{n} A\right) x, x\right\rangle & =\left(1-\beta_{n}\right)\|x\|^{2}-\varepsilon_{n}\langle A x, x\rangle \\
& \geq\left(1-\beta_{n}\right)\|x\|^{2}-\varepsilon_{n}\|A\| \\
& \geq 0 .
\end{aligned}
$$


So this shows that $\left(1-\beta_{n}\right) I-\varepsilon_{n} A$ is positive. It follows that

$$
\begin{aligned}
\left\|\left(1-\beta_{n}\right) I-\varepsilon_{n} A\right\| & =\sup \left\{\left|\left\langle\left(\left(1-\beta_{n}\right) I-\varepsilon_{n} A\right) x, x\right\rangle\right|: x \subseteq H,\|x\|=1\right\} \\
& =\sup \left\{1-\beta_{n}-\varepsilon_{n}\langle A x, x\rangle x \subseteq H,\|x\|=1\right\} \leq 1-\beta_{n}-\varepsilon_{n} \bar{\gamma}
\end{aligned}
$$

Step 1. We claim that the mapping $P_{\Omega}(I-A+\gamma f)$ where $\Omega=\bigcap_{i=1}^{\infty} F\left(S_{i}\right) \cap E P(\Phi)$ has a unique fixed point. Let $f$ be a contraction of $H$ into itself with $\alpha \in(0,1)$. Then, we have

$$
\begin{aligned}
\left\|P_{C}(I-A+\gamma f)(x)-P_{C}(I-A+\gamma f)(y)\right\| & \leq\|(I-A+\gamma f)(x)-(I-A+\gamma f)(y)\| \\
& \leq\|I-A\|\|x-y\|+\gamma\|f(x)-f(y)\| \\
& \leq(1-\bar{\gamma})\|x-y\|+\gamma \alpha\|x-y\| \\
& =(1-(\bar{\gamma}-\gamma \alpha))\|x-y\|,
\end{aligned}
$$

for all $x, y \in H$. Since $0<1-(\bar{\gamma}-\gamma \alpha)<1$, it follows that $P_{\Omega}(I-A+\gamma f)$ is a contraction of $H$ into itself. Therefore the Banach contraction mapping principle implies that there exists a unique element $q \in H$ such that $q=P_{\Omega}(I-A+\gamma f)(q)$.

Step 2 . We shall show that $\left(I-\mu_{n} B\right)$ is nonexpansive. Let $x, y \in C$. Since $B$ is $\xi$-inverse strongly monotone and $\lambda_{n}<2 \alpha$ for all $n \in \mathbb{N}$, we obtain

$$
\begin{aligned}
\left\|\left(I-\mu_{n} B\right) x-\left(I-\mu_{n} B\right) y\right\|^{2}= & \left\|x-y-\mu_{n}(B x-B y)\right\|^{2} \\
= & \|x-y\|^{2}-2 \mu_{n}\langle x-y, B x-B y\rangle \\
& +\mu_{n}^{2}\|B x-B y\|^{2} \\
\leq & \|x-y\|^{2}-2 \xi \mu_{n}\|B x-B y\|^{2}+\mu_{n}^{2}\|B x-B y\|^{2} \\
= & \|x-y\|^{2}+\mu_{n}\left(\mu_{n}-2 \xi\right)\|B x-B y\|^{2} \leq\|x-y\|^{2},
\end{aligned}
$$

where $\mu_{n} \leq 2 \xi$, for all $n \in N$. So we have that the mapping $\left(I-\lambda_{n} A\right)$ is nonexpansive.

Step 3. We claim that $\left\{x_{n}\right\}$ is bounded.

Let $p \in \Omega$; from Lemma 2.10, we have

$$
\begin{gathered}
p=P_{C}\left(p-\mu_{n} B p\right)=T_{\lambda_{n}} p, \\
\left\|z_{n}-p\right\|=\left\|T_{\lambda_{n}} x_{n}-T_{\lambda_{n}} p\right\| \\
\leq\left\|x_{n}-p\right\| .
\end{gathered}
$$


Note that

$$
\begin{aligned}
\left\|y_{n}-p\right\| & =\left\|P_{C}\left(I-\mu_{n} B\right) z_{n}-p\right\| \\
& =\left\|P_{C}\left(I-\mu_{n} B\right) z_{n}-P_{C}\left(I-\mu_{n} B\right) p\right\| \\
& \leq\left\|\left(z_{n}-\mu_{n} B z_{n}\right)-\left(p-\mu_{n} B p\right)\right\| \\
& =\left\|\left(I-\mu_{n} B\right)\left(z_{n}-p\right)\right\| \\
& \leq\left\|z_{n}-p\right\| \\
& \leq\left\|x_{n}-p\right\|, \\
\left\|K_{n}-p\right\| & =\left\|\alpha_{n} x_{n}+\left(1-\alpha_{n}\right) W_{n} y_{n}-p\right\| \\
& =\left\|\alpha_{n}\left(x_{n}-p\right)+\left(1-\alpha_{n}\right)\left(W_{n} y_{n}-p\right)\right\| \\
& \leq \alpha_{n}\left\|x_{n}-p\right\|+\left(1-\alpha_{n}\right)\left\|W_{n} y_{n}-p\right\| \\
& \leq \alpha_{n}\left\|x_{n}-p\right\|+\left(1-\alpha_{n}\right)\left\|y_{n}-p\right\| \\
& \leq\left\|x_{n}-p\right\| .
\end{aligned}
$$

It follows that

$$
\begin{aligned}
\left\|x_{n+1}-p\right\| & =\left\|\varepsilon_{n} \gamma f\left(x_{n}\right)+\beta_{n} x_{n}+\left(\left(1-\beta_{n}\right) I-\varepsilon_{n} A\right) K_{n}-p\right\| \\
& =\left\|\varepsilon_{n}\left(\gamma f\left(x_{n}\right)-A p\right)+\beta_{n}\left(x_{n}-p\right)+\left(\left(1-\beta_{n}\right) I-\varepsilon_{n} A\right)\left(K_{n}-p\right)\right\| \\
& \leq \varepsilon_{n}\left\|\gamma f\left(x_{n}\right)-A p\right\|+\beta_{n}\left\|x_{n}-p\right\|+\left(1-\beta_{n}-\varepsilon_{n} \bar{\gamma}\right)\left\|K_{n}-p\right\| \\
& \leq \varepsilon_{n}\left\|\gamma f\left(x_{n}\right)-A p\right\|+\beta_{n}\left\|x_{n}-p\right\|+\left(1-\beta_{n}-\varepsilon_{n} \bar{\gamma}\right)\left\|x_{n}-p\right\| \\
& \leq\left(1-\varepsilon_{n} \bar{\gamma}\right)\left\|x_{n}-p\right\|+\varepsilon_{n} \gamma\left\|f\left(x_{n}\right)-f(p)\right\|+\varepsilon_{n}\|\gamma f(p)-A p\| \\
& \leq\left(1-\varepsilon_{n} \bar{\gamma}\right)\left\|x_{n}-p\right\|+\varepsilon_{n} \gamma \alpha\left\|x_{n}-p\right\|+\varepsilon_{n}\|\gamma f(p)-A p\| \\
& =\left(1-(\bar{\gamma}-\alpha \gamma) \varepsilon_{n}\right)\left\|x_{n}-p\right\|+(\bar{\gamma}-\alpha \gamma) \varepsilon_{n} \frac{\|\gamma f(p)-A p\|}{\bar{\gamma}-\alpha \gamma} \\
& \leq \max \left\{\left\|x_{n}-p\right\|, \frac{\|\gamma f(p)-A p\|}{\bar{\gamma}-\alpha \gamma}\right\} .
\end{aligned}
$$

By simple induction, we have

$$
\left\|x_{n}-p\right\| \leq \max \left\{\left\|x_{1}-p\right\|, \frac{\|\gamma f(p)-A p\|}{\bar{\gamma}-\alpha \gamma}\right\}, \quad \forall n \in \mathbb{N} .
$$

Hence $\left\{x_{n}\right\}$ is bounded. This implies that $\left\{K_{n}\right\},\left\{f\left(x_{n}\right)\right\}$ are also bounded. 
Step 4. Show that $\limsup _{n \rightarrow \infty}\left\|x_{n+1}-x_{n}\right\|=0$.

Observing that $z_{n}=T_{\lambda_{n}} x_{n}$ and $z_{n+1}=T_{\lambda_{n+1}}$, we get

$$
\begin{aligned}
\left\|z_{n+1}-z_{n}\right\| & =\left\|T_{\lambda_{n+1}} x_{n+1}-T_{\lambda_{n}} x_{n}\right\| \\
& =\left\|T_{\lambda_{n+1}} x_{n+1}-T_{\lambda_{n+1}} x_{n}+T_{\lambda_{n+1}} x_{n}-T_{\lambda_{n}} x_{n}\right\| \\
& \leq\left\|x_{n+1}-x_{n}\right\|+\left\|T_{\lambda_{n+1}} x_{n}-T_{\lambda_{n}} x_{n}\right\| .
\end{aligned}
$$

By Lemma 2.10, we obtain

$$
\begin{gathered}
\Phi\left(T_{\lambda_{n}} x_{n}, y\right)+\frac{1}{\lambda_{n}}\left\langle y-T_{\lambda_{n}} x_{n}, T_{\lambda_{n}} u_{n}-x_{n}\right\rangle \geq 0, \quad \forall y \in C, \\
\Phi\left(T_{\lambda_{n+1}} x_{n}, y\right)+\frac{1}{\lambda_{n+1}}\left\langle y-T_{\lambda_{n+1}} x_{n}, T_{\lambda_{n+1}} x_{n}-x_{n}\right\rangle \geq 0, \quad \forall y \in C .
\end{gathered}
$$

In particular, we have

$$
\begin{gathered}
\Phi\left(T_{\lambda_{n}} x_{n}, T_{\lambda_{n+1}} x_{n}\right)+\frac{1}{\lambda_{n}}\left\langle T_{\lambda_{n+1}} x_{n}-T_{\lambda_{n}} x_{n}, T_{\lambda_{n}} x_{n}-x_{n}\right\rangle \geq 0, \\
\Phi\left(T_{\lambda_{n+1}} x_{n}, T_{\lambda_{n}} x_{n}\right)+\frac{1}{\lambda_{n+1}}\left\langle T_{\lambda_{n}} x_{n}-T_{\lambda_{n+1}} x_{n}, T_{\lambda_{n+1}} x_{n}-x_{n}\right\rangle \geq 0 .
\end{gathered}
$$

Summing up (3.14) and using $\left(\mathrm{A}_{2}\right)$, we obtain

$$
\frac{1}{\lambda_{n+1}}\left\langle T_{\lambda_{n}} x_{n}-T_{\lambda_{n+1}} x_{n}, T_{\lambda_{n+1}} x_{n}-x_{n}\right\rangle+\frac{1}{\lambda_{n}}\left\langle T_{\lambda_{n+1}} x_{n}-T_{\lambda_{n}} x_{n}, T_{\lambda_{n}} x_{n}-x_{n}\right\rangle \geq 0,
$$

for all $y \in C$. It follows that

$$
\left\langle T_{\lambda_{n}} x_{n}-T_{\lambda_{n+1}} x_{n}, \frac{T_{\lambda_{n+1}} x_{n}-x_{n}}{\lambda_{n+1}}-\frac{T_{\lambda_{n}} x_{n}-x_{n}}{\lambda_{n}}\right\rangle \geq 0 .
$$

This implies

$$
\begin{aligned}
0 & \leq\left\langle T_{\lambda_{n+1}} x_{n}-T_{\lambda_{n}} x_{n}, T_{\lambda_{n}} x_{n}-x_{n}-\frac{\lambda_{n}}{\lambda_{n+1}}\left(T_{\lambda_{n+1}} x_{n}-x_{n}\right)\right\rangle \\
& =\left\langle T_{\lambda_{n+1}} x_{n}-T_{\lambda_{n}} x_{n}, T_{\lambda_{n}} x_{n}-T_{\lambda_{n+1}} x_{n}+\left(1-\frac{\lambda_{n}}{\lambda_{n+1}}\right)\left(T_{\lambda_{n+1}} x_{n}-x_{n}\right)\right\rangle
\end{aligned}
$$

It follows that

$$
\left\|T_{\lambda_{n+1}} x_{n}-T_{\lambda_{n}} x_{n}\right\|^{2} \leq\left|1-\frac{\lambda_{n}}{\lambda_{n+1}}\right|\left\|T_{\lambda_{n+1}} x_{n}-T_{\lambda_{n}} x_{n}\right\|\left(\left\|T_{\lambda_{n+1}} x_{n}\right\|+\left\|x_{n}\right\|\right) .
$$


Journal of Applied Mathematics

Hence, we obtain

$$
\left\|T_{\lambda_{n+1}} x_{n}-T_{\lambda_{n}} x_{n}\right\|^{2} \leq\left|1-\frac{\lambda_{n}}{\lambda_{n+1}}\right| L,
$$

where $L=\sup \left\{\left\|x_{n}\right\|+\left\|T_{\lambda_{n+1}} x_{n}\right\|: n \in \mathbb{N}\right\}$. By (3.12) and (3.19), we obtain

$$
\begin{aligned}
\left\|z_{n+1}-z_{n}\right\| & \leq\left\|u_{n+1}-u_{n}\right\|+\left\|T_{\lambda_{n+1}} u_{n}-T_{\lambda_{n}} u_{n}\right\| \\
& \leq\left\|x_{n+1}-x_{n}\right\|+\left|1-\frac{\lambda_{n}}{\lambda_{n+1}}\right| L, \\
\left\|y_{n+1}-y_{n}\right\| & =\left\|P_{C}\left(I-\mu_{n} B\right) z_{n+1}-P_{C}\left(I-\mu_{n} B\right) z_{n}\right\| \\
& \leq\left\|\left(I-\mu_{n} B\right)\left(z_{n+1}-z_{n}\right)\right\| \\
& \leq\left\|z_{n+1}-z_{n}\right\| \leq\left\|x_{n+1}-x_{n}\right\|+\left|1-\frac{\lambda_{n}}{\lambda_{n+1}}\right| L .
\end{aligned}
$$

From (2.5), we have

$$
\begin{aligned}
\left\|W_{n+1} y_{n}-W_{n} y_{n}\right\| & =\left\|t_{1} S_{1}^{\prime} U_{n+1,2} y_{n}-t_{1} S_{1}^{\prime} U_{n, 2} y_{n}\right\| \\
& \leq t_{1}\left\|U_{n+1,2} y_{n}-U_{n, 2}\right\| \\
& \leq t_{1}\left\|t_{2} S_{2}^{\prime} U_{n+1,3} y_{n}-t_{2} S_{2}^{\prime} U_{n, 3} y_{n}\right\| \\
& \leq t_{1} t_{2}\left\|U_{n+1,3} y_{n}-U_{n, 3} y_{n}\right\| \\
& \leq \cdots \\
& \leq M_{1} \prod_{i=1}^{n} t_{i}
\end{aligned}
$$

where $M_{1}=\sup _{n}\left\{\left\|U_{n+1, n+1} y_{n}-U_{n, n+1} y_{n}\right\|\right\}$.

Note that

$$
\begin{aligned}
\left\|K_{n+1}-K_{n}\right\|= & \left\|\alpha_{n+1} x_{n+1}+\left(1-\alpha_{n+1}\right) W_{n+1} y_{n+1}-\alpha_{n} x_{n}-\left(1-\alpha_{n}\right) W_{n} y_{n}\right\| \\
= & \| \alpha_{n+1}\left(x_{n+1}-x_{n}\right)+\alpha_{n+1} x_{n}+\left(1-\alpha_{n+1}\right)\left(W_{n+1} y_{n+1}-W_{n} y_{n}\right) \\
& -\alpha_{n} x_{n}+\left(1-\alpha_{n+1}\right) W_{n} y_{n}-\left(1-\alpha_{n}\right) W_{n} y_{n} \| \\
= & \| \alpha_{n+1}\left(x_{n+1}-x_{n}\right)+\left(\alpha_{n+1}-\alpha_{n}\right) x_{n}\left(1-\alpha_{n+1}\right)\left(W_{n+1} y_{n+1}-W_{n} y_{n}\right) \\
& \quad+\left(\alpha_{n}-\alpha_{n+1}\right) W_{n} y_{n} \|
\end{aligned}
$$




$$
\begin{aligned}
\leq & \alpha_{n+1}\left\|x_{n+1}-x_{n}\right\|+\left|\alpha_{n+1}-\alpha_{n}\right|\left\|x_{n}\right\| \\
& +\left(1-\alpha_{n+1}\right)\left\|W_{n+1} y_{n+1}-W_{n} y_{n}\right\|+\left|\alpha_{n}-\alpha_{n+1}\right|\left\|W_{n} y_{n}\right\| \\
\leq & \alpha_{n+1}\left\|x_{n+1}-x_{n}\right\|+\left|\alpha_{n+1}-\alpha_{n}\right|\left\|x_{n}\right\| \\
& +\left(1-\alpha_{n+1}\right)\left[\left\|W_{n+1} y_{n+1}-W_{n+1} y_{n}\right\|+\left\|W_{n+1} y_{n}-W_{n} y_{n}\right\|\right] \\
& +\left|\alpha_{n}-\alpha_{n+1}\right|\left\|W_{n} y_{n}\right\| \\
\leq & \alpha_{n+1}\left\|x_{n+1}-x_{n}\right\|+\left|\alpha_{n+1}-\alpha_{n}\right|\left\|x_{n}\right\| \\
& +\left(1-\alpha_{n+1}\right)\left[\left\|x_{n+1}-x_{n}\right\|+\left(1-\alpha_{n+1}\right)\left|1-\frac{\lambda_{n}}{\lambda_{n+1}}\right| L+M_{1} \prod_{i=1}^{n} t_{i}\right] \\
& +\left|\alpha_{n}-\alpha_{n+1}\right|\left\|W_{n} y_{n}\right\| \\
\leq & \left\|x_{n+1}-x_{n}\right\|+2\left|\alpha_{n+1}-\alpha_{n}\right| M_{2}^{\prime}+\left(1-\alpha_{n+1}\right)\left(M_{1} \prod_{i=1}^{n} t_{i}+\left|1-\frac{\lambda_{n}}{\lambda_{n+1}}\right| L\right),
\end{aligned}
$$

where $M_{2}^{\prime}=\sup \left\{\left\|x_{n}\right\|,\left\|W_{n} y_{n}\right\|\right\}$.

Suppose $x_{n+1}=\beta_{n} x_{n}+\left(1-\beta_{n}\right) l_{n}$, then $l_{n}=\left(x_{n+1}-\beta_{n} x_{n}\right) /\left(1-\beta_{n}\right)=\left(\varepsilon_{n} \gamma f\left(x_{n}\right)+((1-\right.$ $\left.\left.\left.\beta_{n}\right) I-\varepsilon_{n} F\right) K_{n}\right) /\left(1-\beta_{n}\right)$.

Hence, we have

$$
\begin{aligned}
l_{n+1}-l_{n} & =\frac{\varepsilon_{n+1} \gamma f\left(x_{n+1}\right)+\left(\left(1-\beta_{n+1}\right) I-\varepsilon_{n} A\right) K_{n+1}}{1-\beta_{n+1}}-\frac{\varepsilon_{n} \gamma f\left(x_{n}\right)+\left(\left(1-\beta_{n}\right) I-\varepsilon_{n} A\right) K_{n}}{1-\beta_{n}} \\
& =\frac{\varepsilon_{n+1}}{1-\beta_{n+1}} \gamma f\left(x_{n+1}\right)-\frac{\varepsilon_{n}}{1-\beta_{n}} \gamma f\left(x_{n}\right)+K_{n+1}-K_{n}+\frac{\varepsilon_{n}}{1-\beta_{n}} A K_{n}-\frac{\varepsilon_{n+1}}{1-\beta_{n+1}} A K_{n+1} \\
& =\frac{\varepsilon_{n+1}}{1-\beta_{n+1}}\left(\gamma f\left(x_{n+1}-A K_{n+1}\right)\right)+\frac{\varepsilon_{n}}{1-\beta_{n}}\left(A K_{n}-\gamma f\left(x_{n}\right)\right)+K_{n+1}-K_{n} .
\end{aligned}
$$

Then

$$
\begin{aligned}
\left\|l_{n+1}-l_{n}\right\|-\left\|x_{n+1}-x_{n}\right\| \leq & \frac{\varepsilon_{n+1}}{1-\beta_{n+1}}\left(\left\|\gamma f\left(x_{n+1}\right)\right\|+\left\|A K_{n+1}\right\|\right) \\
& +\frac{\varepsilon_{n}}{1-\beta_{n}}\left(\left\|A K_{n}\right\|+\left\|\gamma f\left(x_{n}\right)\right\|\right)+\left\|K_{n+1}-K_{n}\right\|-\left\|x_{n+1}-x_{n}\right\| \\
\leq & \frac{\varepsilon_{n+1}}{1-\beta_{n+1}}\left(\left\|\gamma f\left(x_{n+1}\right)\right\|+\left\|A K_{n+1}\right\|\right)+\frac{\varepsilon_{n}}{1-\beta_{n}}\left(\left\|A K_{n}\right\|+\left\|\gamma f\left(x_{n}\right)\right\|\right) \\
& +2\left|\alpha_{n+1}-\alpha_{n}\right| M_{2}^{\prime}+\left(1-\alpha_{n+1}\right)\left(\left|1-\frac{\lambda_{n}}{\lambda_{n+1}}\right| L+M_{1} \prod_{i=1}^{n} t_{i}\right) .
\end{aligned}
$$


Journal of Applied Mathematics

Combining with (i), (iii), and (iv), we have

$$
\limsup _{n \rightarrow \infty}\left(\left\|l_{n+1}-l_{n}\right\|-\left\|x_{n+1}-x_{n}\right\|\right) \leq 0
$$

Hence, by Lemma 2.11, we obtain $\left\|l_{n}-x_{n}\right\| \rightarrow 0$ as $n \rightarrow \infty$. It follows that

$$
\lim _{n \rightarrow \infty}\left\|x_{n+1}-x_{n}\right\|=\lim _{n \rightarrow \infty}\left(1-\beta_{n}\right)\left\|l_{n}-x_{n}\right\|=0 .
$$

We also know that

$$
\begin{aligned}
x_{n+1}-x_{n} & =\varepsilon_{n} \gamma f\left(x_{n}\right)+\beta_{n} x_{n}+\left[\left(1-\beta_{n}\right) I-\varepsilon_{n} A\right] K_{n}-x_{n} \\
& =\varepsilon_{n}\left(\gamma f\left(x_{n}\right)-A x_{n}\right)+\left[\left(1-\beta_{n}\right) I-\varepsilon_{n} A\right]\left(K_{n}-x_{n}\right) .
\end{aligned}
$$

So

$$
\lim _{n \rightarrow \infty}\left\|K_{n}-x_{n}\right\|=0
$$

Step 5. We claim that $\left\|x_{n}-W x_{n}\right\| \rightarrow 0$.

Observe that

$$
\begin{aligned}
\left\|x_{n}-W_{n} y_{n}\right\| & =\left\|x_{n}-x_{n+1}+x_{n+1}-y_{n}+K_{n}-W_{n} y_{n}\right\| \\
& \leq\left\|x_{n}-x_{n+1}\right\|+\left\|x_{n+1}-y_{n}\right\|+\alpha_{n}\left\|x_{n}-W_{n} y_{n}\right\| .
\end{aligned}
$$

From (A1), (A3), and (3.28), using step 2, we have

$$
\begin{aligned}
\left(1-\alpha_{n}\right)\left\|x_{n}-W_{n} y_{n}\right\| & \leq\left\|x_{n}-x_{n+1}\right\|+\left\|\varepsilon_{n} \gamma f\left(x_{n}\right)+\beta_{n} x_{n}-\beta_{n} K_{n}-\varepsilon_{n} A K_{n}\right\| \\
& \leq\left\|x_{n}-x_{n+1}\right\|+\varepsilon_{n}\left\|\gamma f\left(x_{n}\right)-A K_{n}\right\|+\beta_{n}\left\|x_{n}-K_{n}\right\| .
\end{aligned}
$$

This implies that

$$
\left\|x_{n}-W_{n} y_{n}\right\| \longrightarrow 0 \quad(\text { as } n \longrightarrow \infty)
$$

Next we want to show $\lim _{n \rightarrow \infty}\left\|x_{n}-y_{n}\right\|=0$.

Let $p \in \bigcap_{i=1}^{\infty} F\left(S_{i}\right) \cap E P(\Phi)$; we have

$$
\begin{aligned}
\left\|z_{n}-p\right\|^{2} & =\left\|T_{\lambda_{n}} x_{n}-T_{\lambda_{n}} p\right\|^{2} \\
& \leq\left\langle T_{\lambda_{n}} x_{n}-T_{\lambda_{n}} p, x_{n}-p\right\rangle \\
& =\left\langle z_{n}-p, x_{n}-p\right\rangle \\
& =\frac{1}{2}\left(\left\|z_{n}-p\right\|^{2}+\left\|x_{n}-p\right\|^{2}-\left\|x_{n}-z_{n}\right\|^{2}\right) .
\end{aligned}
$$


Therefore

$$
\left\|z_{n}-p\right\|^{2} \leq\left\|x_{n}-p\right\|^{2}-\left\|x_{n}-z_{n}\right\|^{2} .
$$

Note that

$$
\begin{aligned}
\left\|K_{n}-p\right\| & =\left\|\alpha_{n} x_{n}+\left(1-\alpha_{n}\right) W_{n} y_{n}-p\right\|^{2} \\
& =\left\|\alpha_{n}\left(x_{n}-p\right)+\left(1-\alpha_{n}\right)\left(W_{n} y_{n}-p\right)\right\|^{2} \\
& \leq \alpha_{n}\left\|x_{n}-p\right\|^{2}+\left(1-\alpha_{n}\right)\left\|y_{n}-p\right\|^{2} \\
& \leq \alpha_{n}\left\|x_{n}-p\right\|^{2}+\left(1-\alpha_{n}\right)\left\|z_{n}-p\right\|^{2} \\
& \leq \alpha_{n}\left\|x_{n}-p\right\|^{2}+\left(1-\alpha_{n}\right)\left[\left\|x_{n}-p\right\|^{2}-\left\|x_{n}-z_{n}\right\|^{2}\right] \\
& =\left\|x_{n}-p\right\|^{2}-\left(1-\alpha_{n}\right)\left\|x_{n}-z_{n}\right\|^{2} .
\end{aligned}
$$

From (3.34), we have

$$
\begin{aligned}
\left\|x_{n+1}-p\right\|^{2}= & \left\|\varepsilon_{n} \gamma f\left(x_{n}\right)+\beta_{n} x_{n}+\left(\left(1-\beta_{n}\right) I-\varepsilon_{n} A\right) K_{n}-p\right\|^{2} \\
= & \left\|\varepsilon_{n}\left(\gamma f\left(x_{n}\right)-A p\right)\right\|^{2}+\beta_{n}\left\|x_{n}-p\right\|^{2}+\left(\left(1-\beta_{n}\right) I-\varepsilon_{n} A\right)\left\|K_{n}-p\right\|^{2} \\
\leq & \varepsilon_{n}\left\|\gamma f\left(x_{n}\right)-A p\right\|^{2}+\beta_{n}\left\|x_{n}-p\right\|^{2}+\left(1-\beta_{n}-\varepsilon_{n} \bar{\gamma}\right)\left\|K_{n}-p\right\|^{2} \\
\leq & \varepsilon_{n}\left\|\gamma f\left(x_{n}\right)-A p\right\|^{2}+\beta_{n}\left\|x_{n}-p\right\|^{2} \\
& +\left(1-\beta_{n}-\varepsilon_{n} \bar{\gamma}\right)\left(\left\|x_{n}-p\right\|^{2}-\left(1-\alpha_{n}\right)\left\|x_{n}-z_{n}\right\|^{2}\right) \\
= & \varepsilon_{n}\left\|\gamma f\left(x_{n}\right)-A p\right\|^{2}+\left(1-\varepsilon_{n} \bar{\gamma}\right)\left\|x_{n}-p\right\|^{2}-\left(1-\beta_{n}-\varepsilon_{n} \bar{\gamma}\right)\left(1-\alpha_{n}\right), \\
\left\|x_{n}-z_{n}\right\|^{2} \leq & \varepsilon_{n}\left\|\gamma f\left(x_{n}\right)-A p\right\|^{2}+\left\|x_{n}-p\right\|^{2}+\left(1-\beta_{n}-\varepsilon_{n} \bar{\gamma}\right)\left(1-\alpha_{n}\right)\left\|x_{n}-z_{n}\right\|^{2} .
\end{aligned}
$$

It follows that

$$
\begin{aligned}
\left(1-\beta_{n}-\varepsilon_{n} \bar{\gamma}\right)\left(1-\alpha_{n}\right)\left\|x_{n}-z_{n}\right\|^{2} \leq & \varepsilon_{n}\left\|\gamma f\left(x_{n}\right)-A p\right\|^{2}+\left\|x_{n}-p\right\|^{2}-\left\|x_{n+1}-p\right\|^{2} \\
= & \varepsilon_{n}\left\|\gamma f\left(x_{n}\right)-A p\right\|^{2}+\left(\left\|x_{n}-p\right\|+\left\|x_{n+1}-p\right\|\right) \\
& \times\left\|x_{n}-x_{n+1}\right\| .
\end{aligned}
$$

From conditions (i), (vi) and (3.26), we have

$$
\left\|x_{n}-z_{n}\right\| \longrightarrow 0 .
$$


We also compute

$$
\begin{aligned}
\left\|y_{n}-p\right\|^{2} & =\left\|P_{C}\left(I-\mu_{n} B\right) z_{n}-P_{C}\left(I-\mu_{n} B\right) p\right\| \\
& \leq\left\|\left(z_{n}-\mu_{n} B z_{n}\right)-\left(p-\mu_{n} B p\right)\right\|^{2} \\
& =\left\|z_{n}-p\right\|^{2}-2 \mu_{n}\left\langle z_{n}-p, B z_{n}-B p\right\rangle+\mu_{n}^{2}\left\|B z_{n}-B p\right\|^{2} \\
& \leq\left\|x_{n}-p\right\|^{2}-\mu_{n}\left(2 \xi-\mu_{n}\right)\left\|B z_{n}-B p\right\|^{2}, \\
\left\|K_{n}-p\right\|^{2} & =\left\|\alpha_{n} x_{n}+\left(1-\alpha_{n}\right) W_{n} y_{n}-p\right\|^{2} \\
& =\left\|\alpha_{n}\left(x_{n}-p\right)-\left(1-\alpha_{n}\right)\left(W_{n} y_{n}-p\right)\right\|^{2} \\
& \leq \alpha_{n}\left\|x_{n}-p\right\|^{2}+\left(1-\alpha_{n}\right)\left\|y_{n}-p\right\|^{2} \\
& =\alpha_{n}\left\|x_{n}-p\right\|^{2}+\left(1-\alpha_{n}\right)\left\{\left\|x_{n}-p\right\|^{2}-\mu_{n}\left(2 \xi-\mu_{n}\right)\left\|B z_{n}-B p\right\|^{2}\right\} \\
& =\left\|x_{n}-p\right\|^{2}-\mu_{n}\left(1-\alpha_{n}\right)\left(2 \xi-\mu_{n}\right)\left\|B z_{n}-B p\right\|^{2} .
\end{aligned}
$$

So, from (3.39), we get

$$
\begin{aligned}
\left\|x_{n+1}-p\right\|^{2}= & \left\|\varepsilon_{n} \gamma f\left(x_{n}\right)+\beta_{n} x_{n}+\left(\left(1-\beta_{n}\right) I-\varepsilon_{n} A\right) K_{n}-p\right\|^{2} \\
= & \left\|\varepsilon_{n}\left(\gamma f\left(x_{n}\right)-A p\right)\right\|^{2}+\beta_{n}\left(x_{n}-p\right)+\left(\left(1-\beta_{n}\right) I-\varepsilon_{n} A\right)\left\|K_{n}-p\right\|^{2} \\
\leq & \varepsilon_{n}\left\|\gamma f\left(x_{n}\right)-A p\right\|^{2}+\beta_{n}\left\|x_{n}-p\right\|^{2}+\left(1-\beta_{n}-\varepsilon_{n} \bar{\gamma}\right)\left\|K_{n}-p\right\|^{2} \\
\leq & \varepsilon_{n}\left\|\gamma f\left(x_{n}\right)-A p\right\|^{2}+\beta_{n}\left\|x_{n}-p\right\|^{2}+\left(1-\beta_{n}-\varepsilon_{n} \bar{\gamma}\right) \\
& \times\left\{\left\|x_{n}-p\right\|^{2}-\mu_{n}\left(1-\alpha_{n}\right)\left(2 \xi-\mu_{n}\right)\left\|B z_{n}-B p\right\|^{2}\right\} \\
\leq & \varepsilon_{n}\left\|\gamma f\left(x_{n}\right)-A p\right\|^{2}+\left\|x_{n}-p\right\|^{2} \\
& -\left(1-\beta_{n}-\varepsilon_{n} \bar{\gamma}\right) \mu_{n}\left(1-\alpha_{n}\right)\left(2 \xi-\mu_{n}\right)\left\|B z_{n}-B p\right\|^{2} .
\end{aligned}
$$

It follows that

$$
\begin{aligned}
& \left(1-\beta_{n}-\varepsilon_{n} \bar{\gamma}\right) \mu_{n}\left(1-\alpha_{n}\right)\left(2 \xi-\mu_{n}\right)\left\|B z_{n}-B p\right\|^{2} \\
& \quad \leq \varepsilon_{n}\left\|\gamma f\left(x_{n}\right)-A p\right\|^{2}+\left\|x_{n}-x_{n+1}\right\|\left(\left\|x_{n}-p\right\|+\left\|x_{n+1}-p\right\|\right) .
\end{aligned}
$$

So

$$
\left\|B z_{n}-B p\right\| \longrightarrow 0 .
$$


On the other hand, we also know that

$$
\begin{aligned}
\left\|y_{n}-p\right\|^{2}= & \left\|P_{C}\left(I-\mu_{n} B\right) z_{n}-P_{C}\left(I-\mu_{n} B\right) p\right\|^{2} \\
\leq & \left\langle\left(I-\mu_{n} B\right) z_{n}-\left(I-\mu_{n} B\right) p, y_{n}-p\right\rangle \\
= & \frac{1}{2}\left\{\left\|\left(I-\mu_{n} B\right) z_{n}-\left(I-\mu_{n} B\right) p\right\|^{2}+\left\|y_{n}-p\right\|^{2}\right. \\
& \left.\quad-\left\|\left(I-\mu_{n} B\right) z_{n}-\left(I-\mu_{n} B\right) p-\left(y_{n}-p\right)\right\|^{2}\right\} \\
\leq & \frac{1}{2}\left\{\left\|x_{n}-p\right\|^{2}+\left\|y_{n}-p\right\|^{2}-\left\|z_{n}-y_{n}\right\|^{2}-\mu_{n}\left\|B z_{n}-B p\right\|^{2}\right. \\
& \left.\quad+2 \mu_{n}\left\langle z_{n}-y_{n}, B z_{n}-B p\right\rangle\right\},
\end{aligned}
$$

and hence

$$
\left\|y_{n}-p\right\|^{2} \leq\left\|x_{n}-p\right\|^{2}-\left\|z_{n}-y_{n}\right\|^{2}+2 \mu_{n}\left\|z_{n}-y_{n}\right\|^{2}\left\|B z_{n}-B p\right\| .
$$

So

$$
\begin{aligned}
\left\|K_{n}-p\right\|^{2}= & \left\|\alpha_{n} x_{n}+\left(1-\alpha_{n}\right) W_{n} y_{n}-p\right\|^{2} \\
\leq & \alpha_{n}\left\|x_{n}-p\right\|^{2}+\left(1-\alpha_{n}\right)\left\{\left\|x_{n}-p\right\|^{2}-\left\|z_{n}-y_{n}\right\|^{2}\right\} \\
& +2 \mu_{n}\left\|z_{n}-y_{n}\right\|\left\|B z_{n}-B p\right\| \\
= & \left\|x_{n}-p\right\|^{2}-\left(1-\alpha_{n}\right)\left\|z_{n}-y_{n}\right\|^{2}+2\left(1-\alpha_{n}\right) \mu_{n}\left\|z_{n}-y_{n}\right\|\left\|B z_{n}-B p\right\|, \\
\left\|x_{n+1}-p\right\|^{2}= & \left\|\varepsilon_{n} \gamma f\left(x_{n}\right)+\beta_{n} x_{n}+\left(\left(1-\beta_{n}\right) I-\varepsilon_{n} A\right) K_{n}-p\right\|^{2} \\
\leq & \varepsilon_{n}\left\|\gamma f\left(x_{n}\right)-A p\right\|^{2}+\beta_{n}\left\|x_{n}-p\right\|^{2}+\left(1-\beta_{n}-\varepsilon_{n} \bar{\gamma}\right)\left\|K_{n}-p\right\|^{2} \\
\leq & \varepsilon_{n}\left\|\gamma f\left(x_{n}\right)-A p\right\|^{2}+\left\|x_{n}-p\right\|^{2}-\left(1-\beta_{n}-\varepsilon_{n} \bar{\gamma}\right)\left(1-\alpha_{n}\right)\left\|z_{n}-y_{n}\right\|^{2} \\
& +2\left(1-\alpha_{n}\right) \mu_{n}\left(1-\beta_{n}-\varepsilon_{n} \bar{\gamma}\right)\left\|z_{n}-y_{n}\right\|\left\|B z_{n}-B p\right\| .
\end{aligned}
$$

Hence

$$
\begin{aligned}
(1- & \left.\beta_{n}-\varepsilon_{n} \bar{\gamma}\right)\left(1-\alpha_{n}\right)\left\|z_{n}-y_{n}\right\|^{2} \\
\leq & \varepsilon_{n}\left\|\gamma f\left(x_{n}\right)-A p\right\|^{2}+2\left(1-\alpha_{n}\right) \mu_{n}\left(1-\beta_{n}-\varepsilon_{n} \bar{\gamma}\right)\left\|z_{n}-y_{n}\right\|\left\|B z_{n}-B p\right\| \\
& +\left\|x_{n}-p\right\|^{2}-\left\|x_{n+1}-p\right\|^{2} \\
\leq & \varepsilon_{n}\left\|\gamma f\left(x_{n}\right)-A p\right\|^{2}+2\left(1-\alpha_{n}\right) \mu_{n}\left(1-\beta_{n}-\varepsilon_{n} \bar{\gamma}\right)\left\|z_{n}-y_{n}\right\|\left\|B z_{n}-B p\right\| \\
& +\left\|x_{n}-x_{n+1}\right\|\left(\left\|x_{n}-p\right\|+\left\|x_{n+1}-p\right\|\right) .
\end{aligned}
$$


From (i), (3.42), and (3.26), we know that

$$
\left\|z_{n}-y_{n}\right\| \longrightarrow 0
$$

From (3.37) and (3.42), we can get

$$
\left\|x_{n}-y_{n}\right\| \longrightarrow 0
$$

On the other hand, we have

$$
\begin{aligned}
\left\|x_{n}-W x_{n}\right\| & \leq\left\|x_{n}-W_{n} y_{n}\right\|+\left\|W_{n} y_{n}-W x_{n}\right\| \\
& \leq\left\|x_{n}-W_{n} y_{n}\right\|+\sup \left\|W_{n} y_{n}-W x_{n}\right\| .
\end{aligned}
$$

By (3.30), (3.49), and using Lemma 2.7, we have

$$
\left\|x_{n}-W x_{n}\right\| \longrightarrow 0
$$

Step 6. We claim that $\limsup _{n \rightarrow \infty}\left\langle(A-\gamma f) q, q-x_{n}\right\rangle \leq 0$, where $q=P_{\Omega}(I-A+\gamma f)(q)$ is the unique solution of $\langle(A-\gamma f) q, x-q\rangle \geq 0$, for all $x \in \Omega$.

Indeed, take a subsequence $\left\{x_{n_{j}}\right\}$ of $\left\{x_{n}\right\}$ such that

$$
\limsup _{n \rightarrow \infty}\left\langle(A-\gamma f) q, q-x_{n}\right\rangle=\limsup _{n \rightarrow \infty}\left\langle(A-\gamma f) q, q-x_{n_{j}}\right\rangle .
$$

Since $\left\{x_{n_{j}}\right\}$ is bounded, there exists a subsequence $\left\{x_{n_{j_{k}}}\right\}$ of $\left\{x_{n_{j}}\right\}$, which converges weakly to $p$; without loss of generality, we can assume $x_{n_{j}} \rightarrow p$ and $W x_{n_{j}} \rightarrow p$, we arrive at

$$
\limsup _{n \rightarrow \infty}\left\langle(A-\gamma f) q, q-x_{n_{j}}\right\rangle=\langle(A-\gamma f) q, q-p\rangle \leq 0
$$

Step 7. We show that $x_{n} \rightarrow q$.

Since

$$
\begin{aligned}
\left\langle(A-\gamma f) q, q-x_{n+1}\right\rangle & =\left\langle(A-\gamma f) q, x_{n}-x_{n+1}\right\rangle+\left\langle(A-\gamma f) q, q-x_{n}\right\rangle \\
& \leq\|(A-\gamma f) q\| \cdot\left\|x_{n}-x_{n+1}\right\|+\left\langle(A-\gamma f) q, q-x_{n}\right\rangle,
\end{aligned}
$$

so

$$
\limsup _{n \rightarrow \infty}\left\langle(A-\gamma f) q, q-x_{n+1}\right\rangle \leq 0
$$


Note that

$$
\begin{aligned}
& \left\|x_{n+1}-q\right\|^{2}=\left\|\varepsilon_{n} \gamma f\left(x_{n}\right)+\beta_{n} x_{n}+\left(\left(1-\beta_{n}\right) I-\varepsilon_{n} A\right) K_{n}-q\right\|^{2} \\
& =\left\|\varepsilon_{n}\left(\gamma f\left(x_{n}\right)-A q\right)+\beta_{n}\left(x_{n}-q\right)+\left(\left(1-\beta_{n}\right) I-\varepsilon_{n} A\right)\left(K_{n}-q\right)\right\|^{2} \\
& \leq\left\|\left(1-\beta_{n}\right) \frac{\left(1-\beta_{n}\right) I-\varepsilon_{n} A}{1-\beta_{n}}\left(K_{n}-q\right)+\beta_{n}\left(x_{n}-q\right)\right\|^{2} \\
& +2 \varepsilon_{n}\left\langle(A-\gamma f) q, x_{n+1}-q\right\rangle \\
& \leq\left(1-\beta_{n}\right)\left\|\frac{\left(1-\beta_{n}\right) I-\varepsilon_{n} A}{1-\beta_{n}}\left(K_{n}-q\right)\right\|^{2}+\beta_{n}\left\|x_{n}-q\right\|^{2}+2 \varepsilon_{n} \gamma \alpha \\
& \times\left\langle f\left(x_{n}\right)-f(q), x_{n+1}-q\right\rangle+2 \varepsilon_{n}\left\langle\gamma f(q)-A q, x_{n+1}-q\right\rangle \\
& \leq\left(1-\beta_{n}\right)\left\|\frac{\left(1-\beta_{n}\right) I-\varepsilon_{n} A}{1-\beta_{n}}\left(K_{n}-q\right)\right\|^{2}+\beta_{n}\left\|x_{n}-q\right\|^{2}+2 \varepsilon_{n} \gamma \alpha \\
& \times\left\|x_{n}-q\right\| \cdot\left\|x_{n+1}-q\right\|+2 \varepsilon_{n}\left\langle\gamma f(q)-A q, x_{n+1}-q\right\rangle \\
& \leq \frac{\left\|\left(1-\beta_{n}\right) I-\varepsilon_{n} A\right\|^{2}}{1-\beta_{n}}\left\|K_{n}-q\right\|^{2}+\beta_{n}\left\|x_{n}-q\right\|^{2}+\varepsilon_{n} \gamma \alpha \\
& \times\left(\left\|x_{n}-q\right\|^{2}+\left\|x_{n+1}-q\right\|^{2}\right)+2 \varepsilon_{n}\left\langle\gamma f(q)-A q, x_{n+1}-q\right\rangle \\
& \leq\left(\frac{\left(\left(1-\beta_{n}\right)-\bar{\gamma} \varepsilon_{n}\right)^{2}}{1-\beta_{n}}+\beta_{n}+\varepsilon_{n} \gamma \alpha\right)\left\|x_{n}-q\right\|^{2}+\varepsilon_{n} \gamma \alpha\left\|x_{n+1}-q\right\|^{2} \\
& +2 \varepsilon_{n}\left\langle\gamma f\left(x_{n}\right)-A q, x_{n+1}-q\right\rangle,
\end{aligned}
$$

which implies that

$$
\begin{aligned}
\left\|x_{n+1}-q\right\|^{2} \leq & \left(1-\frac{2(\bar{\gamma}-\alpha \gamma) \varepsilon_{n}}{1-\alpha \gamma \varepsilon_{n}}\right)\left\|x_{n}-q\right\|^{2} \\
& +\frac{\varepsilon_{n}}{1-\alpha \gamma \varepsilon_{n}}\left\{\frac{\bar{\gamma}^{2} \varepsilon_{n}^{2}}{1-\beta_{n}}\left\|x_{n}-q\right\|^{2}+2 \varepsilon_{n}\left\langle\gamma f\left(x_{n}\right)-A q, x_{n+1}-q\right\rangle\right\} .
\end{aligned}
$$

Let

$$
\begin{gathered}
\sigma_{n}=\frac{\varepsilon_{n}}{1-\alpha \gamma \varepsilon_{n}}\left\{\frac{\bar{\gamma}^{2} \varepsilon_{n}^{2}}{1-\beta_{n}}\left\|x_{n}-q\right\|^{2}+2 \varepsilon_{n}\left\langle\gamma f\left(x_{n}\right)-A q, x_{n+1}-q\right\rangle\right\}, \\
\rho_{n}=\frac{2(\bar{\gamma}-\alpha \gamma) \varepsilon_{n}}{1-\alpha \gamma \varepsilon_{n}}
\end{gathered}
$$


then we have

$$
\limsup _{n \rightarrow \infty} \frac{\sigma_{n}}{\rho_{n}} \leq 0
$$

Applying Lemma 2.2, we can conclude that $\left\{x_{n}\right\}$ converges strongly to $q$ in norm. This completes the proof.

As direct consequences of Theorem 3.1, we obtain the following corollary.

Corollary 3.2. Let $C$ be a nonempty closed convex subset of a real Hilbert space $H$. Let $F$ be a bifunction from $C \times C \rightarrow \mathbb{R}$ satisfying (A1)-(A4). Let $S_{i}: C \rightarrow C$ be a family $\kappa_{i}$-strict pseudocontractions for some $0 \leq \kappa_{i}<1$. Assume the set $\Omega=\cap_{i=1}^{\infty} F\left(S_{i}\right) \cap E P(F) \neq \emptyset$. Let $f$ be a contraction of $H$ into itself with $\alpha \in(0,1)$ and let $A$ be an $\alpha$-inverse strongly monotone mapping. Let $F$ be a strongly positive linear-bounded operator on $H$ with coefficient $\gamma>0$ and $0<\gamma<\bar{\gamma} / \alpha$ and $\tau<1$. Let $W_{n}$ be the mapping generated by $S_{i}^{\prime}$ and $t_{i}$, where $S_{i}: C \rightarrow C$ is a nonexpansive mapping with a fixed point. Let $\left\{x_{n}\right\}$ and $\left\{u_{n}\right\}$ be sequences generated by the following algorithm:

$$
\begin{gathered}
F\left(z_{n}, y\right)+\frac{1}{\lambda_{n}}\left\langle y-z_{n}, z_{n}-x_{n}\right\rangle \geq 0, \\
K_{n}=\alpha_{n} x_{n}+\left(1-\alpha_{n}\right) W_{n} z_{n}, \\
x_{n+1}=\varepsilon_{n} \gamma f\left(x_{n}\right)+\beta_{n} x_{n}+\left(\left(1-\beta_{n}\right) I-\varepsilon_{n} A\right) K_{n},
\end{gathered}
$$

where $\left\{\varepsilon_{n}\right\},\left\{\beta_{n}\right\},\left\{\alpha_{n}\right\}$, and $\left\{\lambda_{n}\right\}$ are sequences in $(0,1)$. Assume that the control sequences satisfy the following restrictions:

(i) $\lim _{n \rightarrow \infty} \varepsilon_{n}=0$ and $\sum_{n=1}^{\infty} \varepsilon_{n}=\infty$;

(ii) $0<\liminf _{n \rightarrow \infty} \beta_{n} \leq \limsup \sup _{n \rightarrow \infty} \beta_{n}<1$;

(iii) $0<\liminf _{n \rightarrow \infty} \lambda_{n} \leq \limsup _{n \rightarrow \infty} \lambda_{n}<1$;

(iv) $\lim _{n \rightarrow \infty}\left|\lambda_{n+1}-\lambda_{n}\right|=\lim _{n \rightarrow \infty}\left|\alpha_{n+1}-\alpha_{n}\right|=0$;

(v) $0<t_{n} \leq b<1$;

(vi) $\lambda_{n}<2 \alpha$.

Then $\left\{x_{n}\right\}$ converges strongly to $w \in \Omega$ where $w=P_{\Omega}(I-A+\gamma f) w$.

\section{Acknowledgments}

This research is supported by the Science Research Foundation Program in the Civil Aviation University of China (07kys08) and the Fundamental Research Funds for the Science of the Central Universities (program no. ZXH2012K001).

\section{References}

[1] F. E. Browder and W. V. Petryshyn, "Construction of fixed points of nonlinear mappings in Hilbert space," Journal of Mathematical Analysis and Applications, vol. 20, pp. 197-228, 1967. 
[2] E. Blum and W. Oettli, "From optimization and variational inequalities to equilibrium problems," The Mathematics Student, vol. 63, no. 1-4, pp. 123-145, 1994.

[3] S.-s. Chang, H. W. Joseph Lee, and C. K. Chan, "A new method for solving equilibrium problem fixed point problem and variational inequality problem with application to optimization," Nonlinear Analysis, vol. 70, no. 9, pp. 3307-3319, 2009.

[4] P. L. Combettes and S. A. Hirstoaga, "Equilibrium programming in Hilbert spaces," Journal of Nonlinear and Convex Analysis, vol. 6, no. 1, pp. 117-136, 2005.

[5] Y. Liu, "A general iterative method for equilibrium problems and strict pseudo-contractions in Hilbert spaces," Nonlinear Analysis, vol. 71, no. 10, pp. 4852-4861, 2009.

[6] W. R. Mann, "Mean value methods in iteration," Proceedings of the American Mathematical Society, vol. 4, pp. 506-510, 1953.

[7] G. Marino and H.-K. Xu, "Weak and strong convergence theorems for strict pseudo-contractions in Hilbert spaces," Journal of Mathematical Analysis and Applications, vol. 329, no. 1, pp. 336-346, 2007.

[8] K. Shimoji and W. Takahashi, "Strong convergence to common fixed points of infinite nonexpansive mappings and applications," Taiwanese Journal of Mathematics, vol. 5, no. 2, pp. 387-404, 2001.

[9] T. Suzuki, "Strong convergence of Krasnoselskii and Mann's type sequences for one-parameter nonexpansive semigroups without Bochner integrals," Journal of Mathematical Analysis and Applications, vol. 305, no. 1, pp. 227-239, 2005.

[10] M. Tian, "A general iterative algorithm for nonexpansive mappings in Hilbert spaces," Nonlinear Analysis, vol. 73, no. 3, pp. 689-694, 2010.

[11] S. Takahashi and W. Takahashi, "Strong convergence theorem for a generalized equilibrium problem and a nonexpansive mapping in a Hilbert space," Nonlinear Analysis, vol. 69, no. 3, pp. 1025-1033, 2008.

[12] G. L. Acedo and H.-K. Xu, "Iterative methods for strict pseudo-contractions in Hilbert spaces," Nonlinear Analysis, vol. 67, no. 7, pp. 2258-2271, 2007.

[13] S. Wang, "A general iterative method for obtaining an infinite family of strictly pseudo-contractive mappings in Hilbert spaces," Applied Mathematics Letters, vol. 24, no. 6, pp. 901-907, 2011.

[14] P. Kumam and C. Jaiboon, "Approximation of common solutions to system of mixed equilibrium problems, variational inequality problem, and strict pseudo-contractive mappings," Fixed Point Theory and Applications, vol. 2011, Article ID 347204, 30 pages, 2011.

[15] P. Kumam, N. Petrot, and R. Wangkeeree, "A hybrid iterative scheme for equilibrium problems and fixed point problems of asymptotically $k$-strict pseudo-contractions," Journal of Computational and Applied Mathematics, vol. 233, no. 8, pp. 2013-2026, 2010.

[16] C. Jaiboon and P. Kumam, "Strong convergence theorems for solving equilibrium problems and fixed point problems of $\xi$-strict pseudo-contraction mappings by two hybrid projection methods," Journal of Computational and Applied Mathematics, vol. 234, no. 3, pp. 722-732, 2010.

[17] U. Inprasit, "Viscosity approximation methods for generalized equilibrium problems and fixed point problems of finite family of nonexpansive mappings in Hilbert spaces," Thai Journal of Mathematics, vol. 8, no. 3, pp. 607-626, 2010.

[18] H. K. Xu, "An iterative approach to quadratic optimization," Journal of Optimization Theory and Applications, vol. 116, no. 3, pp. 659-678, 2003. 


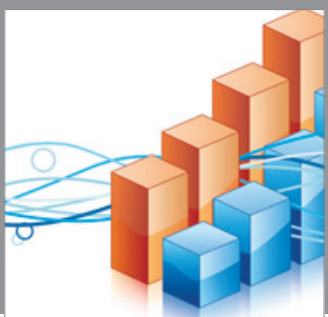

Advances in

Operations Research

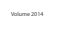

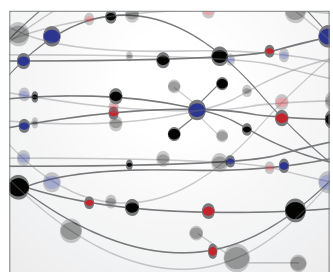

\section{The Scientific} World Journal
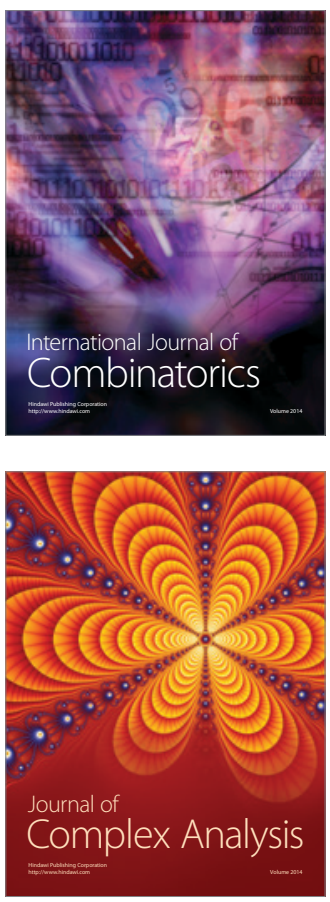

International Journal of

Mathematics and

Mathematical

Sciences
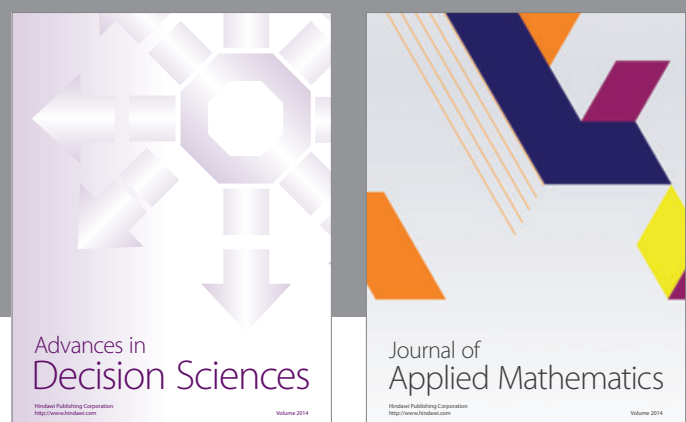

Journal of

Applied Mathematics
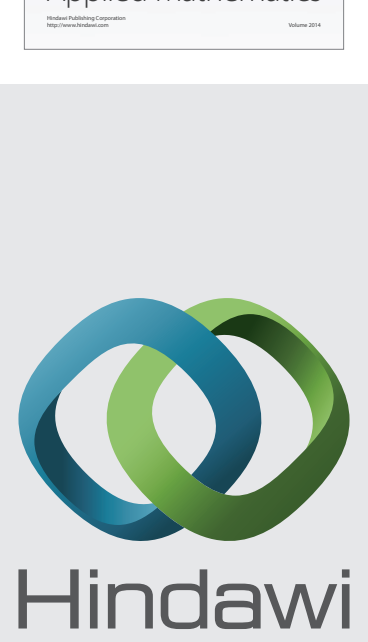

Submit your manuscripts at http://www.hindawi.com
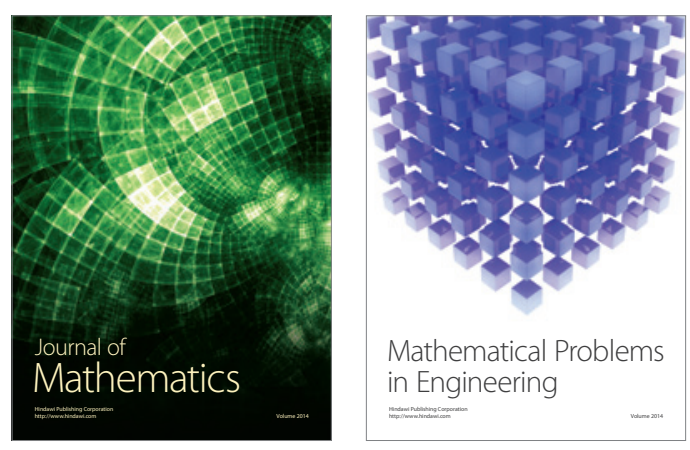

Mathematical Problems in Engineering
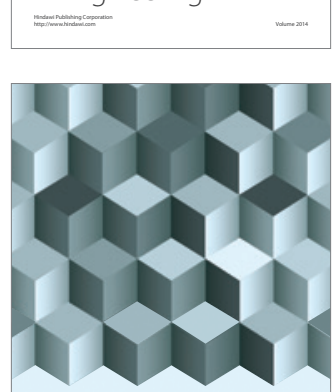

Journal of

Function Spaces
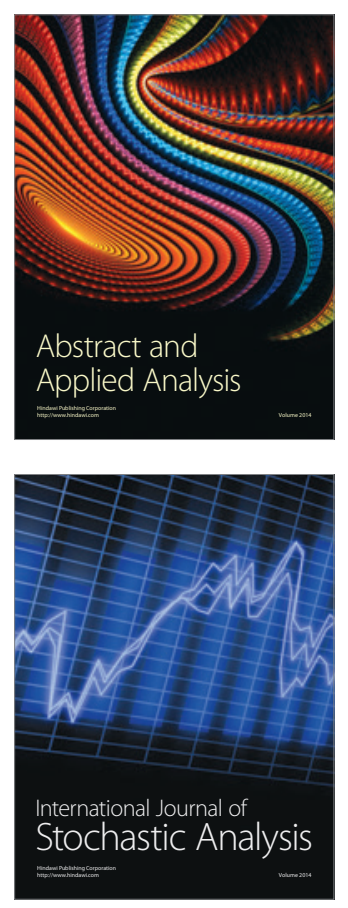

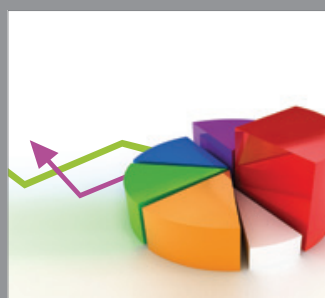

ournal of

Probability and Statistics

Promensencen
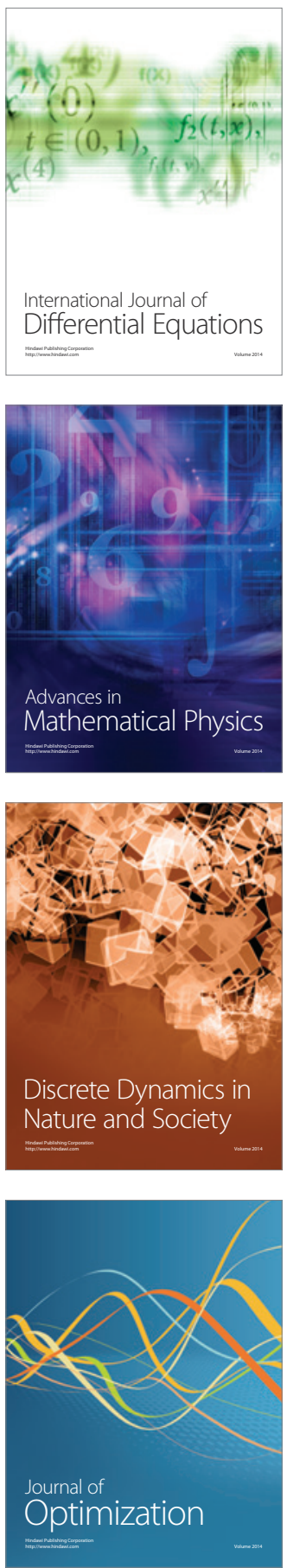